\title{
Membrane InP Saturable Absorbers on Silicon as Building Blocks for Transparent Optical Networks
}

\author{
O. $\operatorname{Raz}^{(1)}$, M. Tassaert ${ }^{(2)}$, G. Roelkens ${ }^{(1,2)}$, H.J.S. Dorren ${ }^{(1)}$ \\ (1) Eindhoven University of Technology, Den Dolech 2, Eindhoven, The Netherlands \\ (2) INTEC, Ghent University-IMEC, St-Pietersnieuwstraat 41, 9000 Ghent, Belgium \\ E-mail:o.raz@tue.nl
}

\begin{abstract}
As silicon photonics continues to gain research and industrial relevance, some of the building blocks in this technology such as modulators and switches still suffer from limitation when it comes to insertion losses and/or extinction ratio. In the past two years we have been investigating a promising new building block for silicon on insulator (SOI) circuits which is based on ultra-thin narrow stripes of InP bonded on top of SOI, which operate as saturable absorbers at very low optical power levels. The unique properties of these membrane InP switches (MIPS) make them ideally suitable as complementary components to SOI devices. By controlling the band gap of the active layers in the membrane as well as the confinement of light critical properties of such MIPS can be determined and tailored to specific applications.

In this talk we will highlight two exciting applications of MIPS on SOI. First we will show how they enhance the performance of silicon based modulators by regenerating the signal through their non-linear response. Secondly we will show how they may solve a unique engineering challenge for next generation access networks by operating as optically controlled switches with high extinction ratio and no electrical power supply.

Keywords: silicon photonics, membrane on silicon devices, active remote nodes for access networks, saturable absorbers, signal regeneration, all optical switches
\end{abstract}

\section{INTRODUCTION}

Photonics has, over the past decades, slowly but surely conquered all transmission distances from transoceanic telecommunications multi $\mathrm{Tb} / \mathrm{sec}$ glass fiber networks to in-car low speed polymer fiber connections. While the clear advantages of signal transmission over dielectric waveguides has made this choice inevitable, data/voice switching has remained almost solely in the electronic domain. The motivation to move from electronic to photonic switches is clear, however optical solutions often suffer from critical drawbacks. Optical switch architectures relaying on broadcast and select solutions are either very lossy or rely on multiple amplification stages degrading the signal to noise ratio. Other solutions which are based on space switching are either too slow (in the case of mechanical or MEMS devices) or show limited switch isolation. In both cases, not reverting to the electronic domain, implies that the signal is not regenerated, meaning a build-up of noise and a degradation of the eye opening.

Silicon photonics is a promising platform for the integration of complex optical circuits as it builds on wellestablished fabrication technology developed for the micro-electronics industry. Although silicon is an excellent material to achieve passive optical functions, the realization of active functions is more challenging due to silicon's indirect band gap. Using heterogeneous integration of III-V on silicon, light sources and detectors have already been successfully demonstrated [1]. Also modulators and switches have been demonstrated in SOI platform but they require large structures or high voltages for operation as the electro-optic effect in silicon is limited [2]. If SOI technology is to emerge as a winning solution for photonic integrated circuits, improved switches and modulators with better properties need to be integrated.

In this paper we show how the performance of both silicon modulators as well as SOI based switches can be greatly improved by adding ultra-thin membrane InP devices. Such membrane InP switches (MIPS) which act as saturable absorbers can acts as both signal regenerators as well as optically controlled high extinction ratio switches.

\section{THE BASIC MIPS DEVICE PROPERTIES AND FABRICATION}

The device layout of the MIPS is schematically shown in Fig. 1(Left). The basic layout consists of a $100 \mathrm{~nm}$ thick InP membrane rib waveguide coupled to a $220 \mathrm{~nm}$ thick underlying silicon circuit using two $18 \mu \mathrm{m}$ long inverted taper couplers to ensure adiabatic coupling of the fundamental TE-mode. The quantum well (QW) stack has three $10 \mathrm{~nm}$ InGaAs QWs are used separated by InP barrier layers $8 \mathrm{~nm}$ thick. The exact control of the band gap of the QWs allows tailoring the spectral response of the MIPS device. For most of the applications discussed in this paper, the MIPS demonstrated high absorption for wavelength below $1600 \mathrm{~nm}$. The width of the membrane rib waveguide is also an important design parameter. Lower widths correspond to lower quantum well volumes and support therefore more efficient pumping, while at the same time surface recombination becomes more important and therefore the carrier lifetime is reduced. For the results described in this paper the 
width was chosen to be $1 \mu \mathrm{m}$. Lower carrier lifetime is critical for operation of the MIPS as a regenerator as will be discussed below. For the use as all-optical switch, also "slower" operation ( $\sim 1 \mathrm{~ns})$ is more than acceptable.
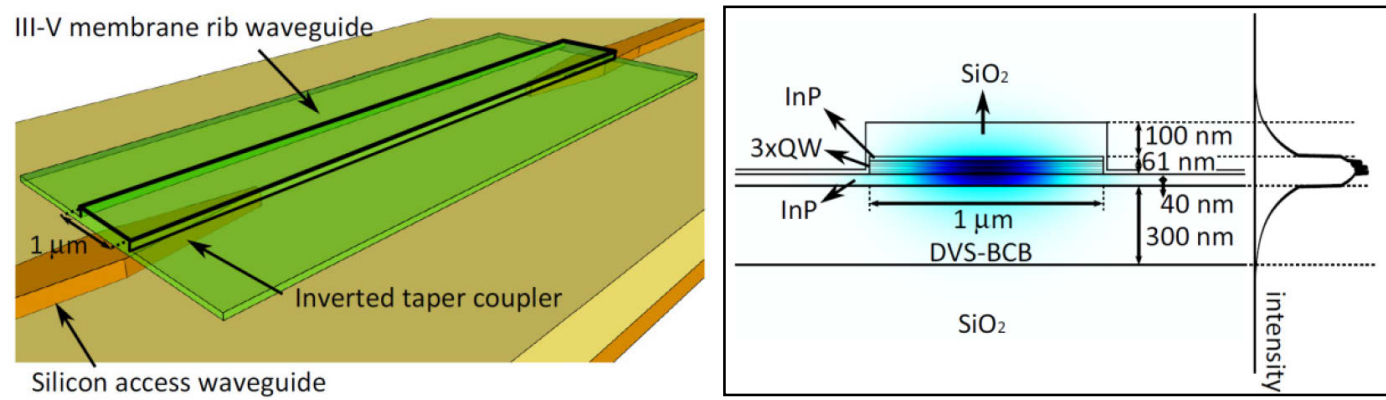

Figure 1: (Left) Schematic view of the device; (Right) Calculated mode profile in the device and cross section of the intensity in the center of the device.

The mode profile in the membrane rib waveguide is shown in Fig. 1(Right). The high confinement makes the MIPS ideal for use as a saturable absorber. This is the case because the differential modal absorption $\partial \alpha / \partial N$ of the device is proportional to the confinement inside the QWs. Therefore, the device absorption will rapidly change with changing carrier concentration $(N)$. In turn, these carriers are created by the absorption of the signal beam itself, which is again proportional to the confinement in the QWs.

\section{THE MIPS REGENERATOR $[3,4]$}

In Fig. 2(Left) the CW device transmission curves are shown for different wavelengths across the C-band. In these transmission curves, three domains can be identified. For low signal powers, there is a quasi-linear relationship between input and output power, as the amount of generated carriers is insufficient to significantly change the high device absorption $\left(\alpha\left(P_{i n}\right) \approx \alpha_{0}\right)$. Also for high signal powers the transmission curves show this proportional relationship, as the amount of generated carriers is sufficient to bleach the absorption completely $\left(\alpha\left(P_{i n}\right) \approx 0\right)$. For moderate powers however, there is a transition between these two regions, which leads to a region where the slope of the transmission curve $\left(\partial P_{\text {out }} / \partial P_{\text {in }}\right)$ is higher than one and values of more than a factor of four are achieved. In this transition region, signal regeneration is possible.
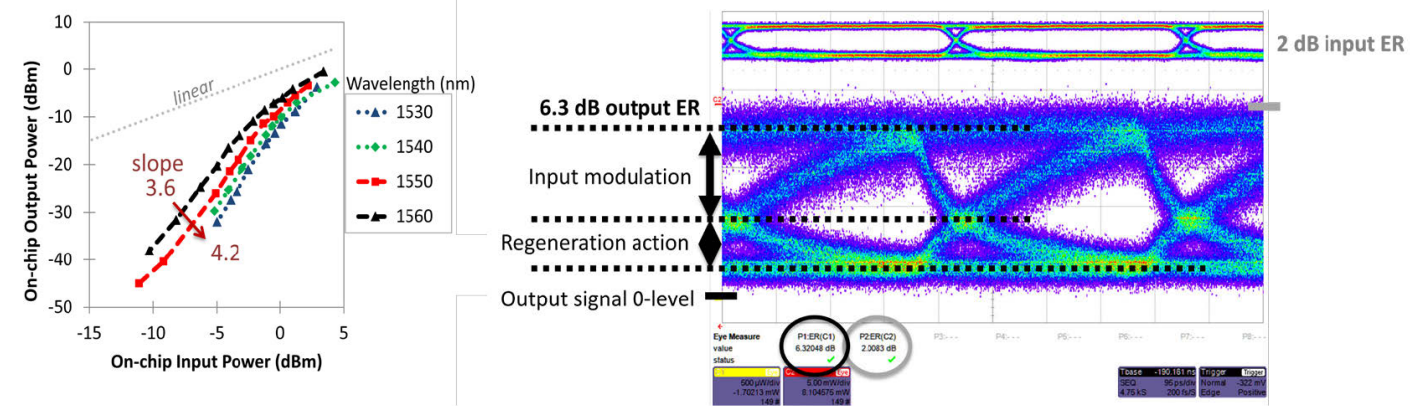

Figure 2: (Left) CW transmission through the device; (Right) Eye diagram: the top eye is the input signal, the bottom eye is the regenerated output signal. The device draws the signal zero-level closer to the real zero-level.

\section{REGENERATOR PERFORMANCE}

To test the regenerator performance, a bit error rate (BER) measurement was performed at a speed of $2.5 \mathrm{~Gb} / \mathrm{s}$. In this measurement a pseudorandom bit sequence of $2^{31}-1$ bits was imprinted on a $\mathrm{CW}$ signal carrier using nonreturn to zero on-off keying (NRZ-OOK) with an ER of only $2 \mathrm{~dB}$. As can be derived from the CW device characteristics, the regeneration performance will depend on the input power of the signal. Therefore, in a first step the optimal working point for regeneration needs to be determined as a function of wavelength. To do this, the signal is sent through the chip at different input powers and the resulting ER of the output signal is recorded by measuring the eye pattern. Furthermore, by comparing the measured output power to a reference measurement the insertion loss of the device is determined. In Fig. 3(Left) the ER and insertion loss are shown for a signal wavelength of $1530 \mathrm{~nm}$ and $1560 \mathrm{~nm}$, and one corresponding eye diagram is shown in Fig. 2(Right).

Unfortunately, there is a trade-off between the regeneration performance of the device and the insertion loss. To avoid high insertion losses, a high power should be applied to bleach the device absorption. However in order to have good regeneration action, the input power should be lower, such that the working point lies in the region with the slope is high $\left(\partial \mathrm{P}_{\text {out }} / \partial \mathrm{P}_{\text {in }}>1\right)$. Next to this, it can be seen that the device performs well over the entire C-band. The results from the BER measurements are shown in Fig. 3(Right). As a working point, we have 
chosen for an optimal regeneration with an insertion loss of $9 \mathrm{~dB}$ for all wavelengths. Compared to the back-toback (B2B) measurement, this yields a receiver sensitivity improvement of over $3.6 \mathrm{~dB}$ over the entire C-band up to $4.5 \mathrm{~dB}$ for the signal at a wavelength of $1530 \mathrm{~nm}$ and $1540 \mathrm{~nm}$.
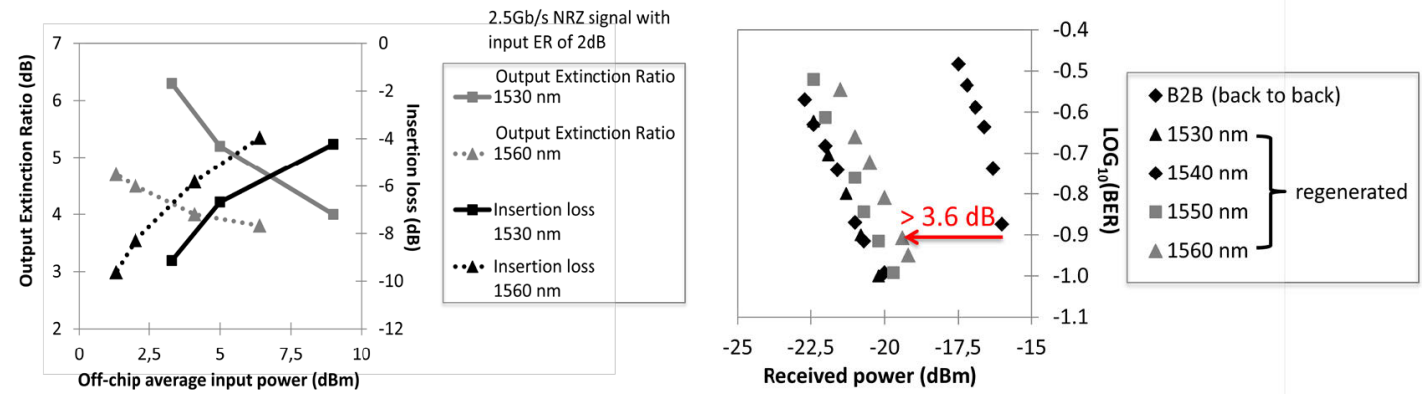

Figure 3: (Left) Output ER and insertion loss as a function of signal input power; (Right) BER measurement as a function of received power.

\section{THE MIPS BASED ACTIVE REMOTE NODE $[5,6,7,8]$}

The $1 \times 4$ switch is based on a silicon-on-insulator circuit fabricated using a multi-project wafer run carried out by ePIXfab. An image of the complete IC is given in Fig. 4.

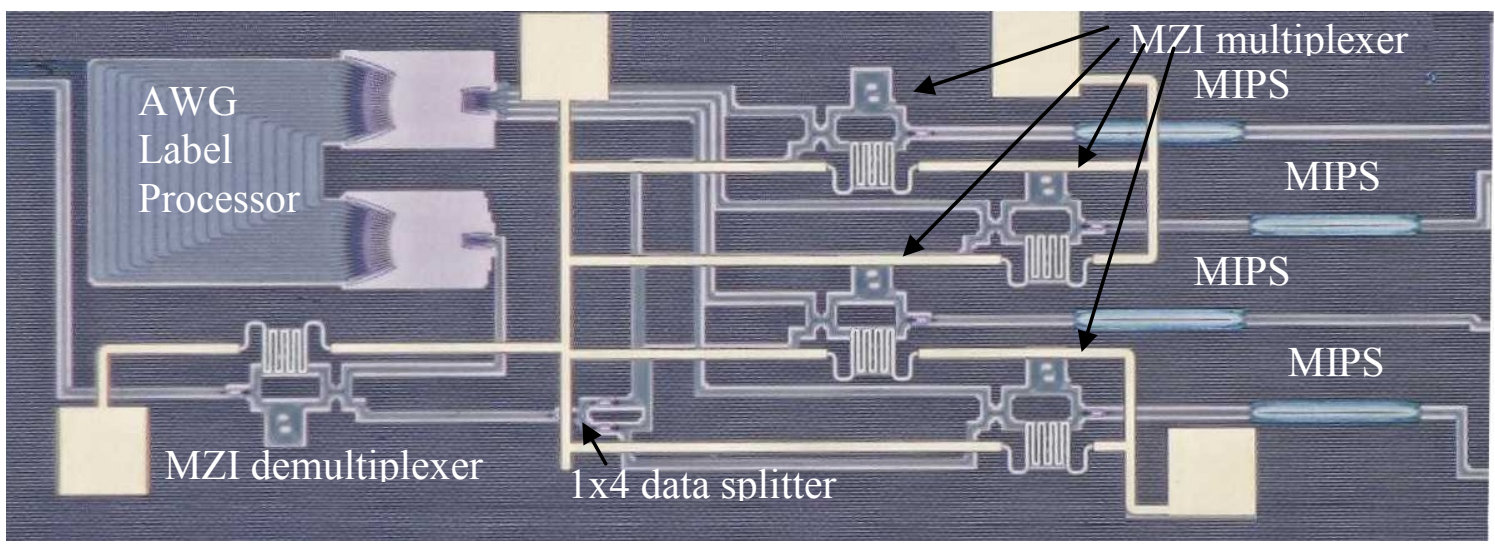

Figure 4. Microscope image of the integrated 1x4 optically controlled remote switch.

This silicon chip is further post processed to include the membrane InP switches, SU-8 inverted tapers and heaters as previously described [6]. The incoming input signal which contains the data and WDM labels is first demultiplexed using a Mach Zehnder interferometer (MZI) into the data path (bottom of chip) and label path (top of the chip). The filtered out data signal is split into four by two consecutive $1 \times 2 \mathrm{MMI}$ splitter stages with each output of the splitter connecting to a separate chip output path. The WDM labels, which were demultiplexed from the data signal, are sent to a $200 \mathrm{GHz}$ channel spacing arrayed waveguide grating filter (AWG). After filtering, the four AWG outputs are multiplexed with the four copies of the data signal using an MZI multiplexer and sent to 4 separate membrane InP switches. The choice of active outputs is done by controlling which of the 4 WDM labels are active when the data packet arrives at the switches. The architecture allows for both unicast, multicast and broadcast operation as no space switching is involved.

\section{THE SWITCH PERFORMANCE}

The full chip was statically characterized for the extinction ratio (ER) of each output when the labels for other channels were present. This is to check for both the intrinsic ER of the membrane switch between the on and off states and the effect of the AWG cross talk on the eventual switch cross talk. In Fig. 5 we show the behavior of a single output of the switch (port number 2) while alternating the label wavelength as well as the ER for the other 3 ports for the on and off states. In this these experiments the label power was set to $0 \mathrm{dBm}$ and the data signal was $8.5 \mathrm{dBm}$ (giving a total insertion loss $>35 \mathrm{~dB}$ ). In Fig. 6 we present the measured BER for a PRBS data stream routed through each one of the output ports while keeping the label operating at $\mathrm{CW}$. The receiver used for these measurement was an AC coupled APD receiver housed inside an XFP module with a typical Rx sensitivity of $-28 \mathrm{dBm}$. Since it was AC coupled it could not be used to measure the packetized BER. 

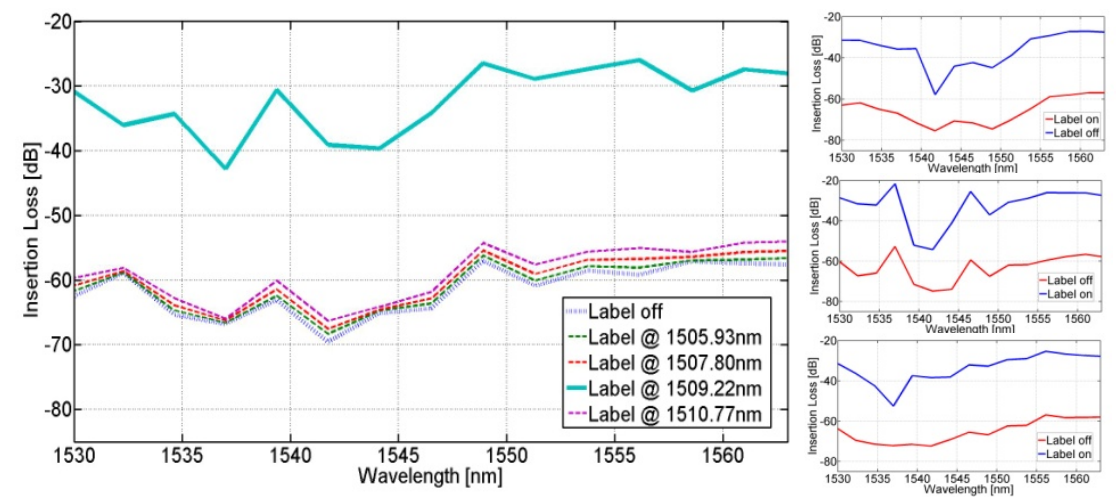

Figure 5: (Left) Port 2 extinction ratio for labels on and off and in the presence of other labels; (Right) Port 1, 3 \& 4 extinction ratio with and without a label present.

For packet transmission we measured BER with an Agilent 8192A $10 \mathrm{~Gb} / \mathrm{s}$ receiver and obtained error free operation with a penalty of $0.8 \mathrm{~dB}$. In addition we show a time trace and eye patterns of the output of the port number 1 when the label is modulated to be on for only one of the 4 packets being transmitted.
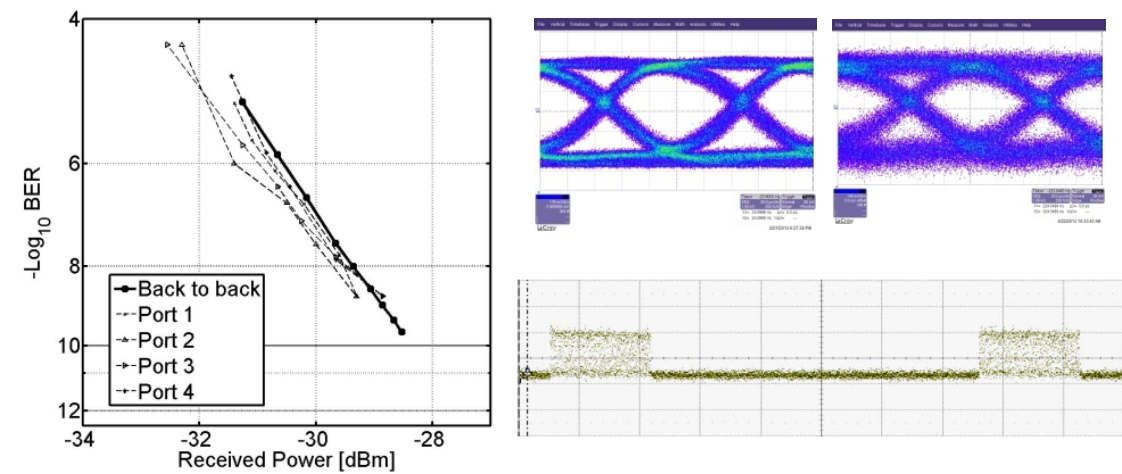

Figure 6: (Left) Measured BER for $10 \mathrm{~Gb} / \mathrm{s}$ PRBS data through the $1 \times 4$ switch for all 4 outputs; (Right, top) Obtained eye patterns for packetized data before and after the switch; (Right, Bottom) Output of port 1 when switching one of 4 packets through it.

The receiver sensitivity after the device shows no measureable penalty for PRBS operation and the chip output shows indeed that the high ER of the MIPS in combination with the high ER of the SOA as label generator insures no data appearing at port 1 output when no label is present. The output eye pattern has been captured with the Agilent DC coupled receiver and is dominated by the electrical noise due to considerably weaker input optical signal.

\section{CONCLUSIONS}

The membrane InP switch or MIPS is an important addition to the set of devices which can be added to passive SOI circuits to increase their functionality. Having no need for electrical connectivity and supporting control of its absorption strength and spectrum through careful epitaxial design and processing, the MIPS can be used both as a very fast saturable absorber for regenerating low ER signals as well as an optically controlled high extinction ratio switch for active remote nodes in future access networks.

\section{REFERENCES}

[1] G. Roelkens et al., Laser Photon. Rev. 4, 751-779 (2010).

[2] G. Reed et al., Nat. Photonics 4, 518-526 (2010).

[3] M. Tassaert et al., Opt. Express 20, 11383-11388 (2012).

[4] M. Tassaert et al., in Proc. OFC 2013, OW3D.4 (2013).

[5] O. Raz et al., in Proc. ECOC 2011, PDP 1.1 (2011).

[6] O. Raz et al., in Proc. ACP 2012, AS1B.5 (2012).

[7] M. Tassaert et al., in Proc. OFC/NFOEC 2013, OW3D4 (2013).

[8] O. Raz et al., in Proc. OFC/NFOEC 2013, PDP5C.2 (2013). 